PROCEEDINGS OF THE AMERICAN MATHEMATICAL SOCIETY

Volume 127, Number 3, March 1999, Pages 813-818

S 0002-9939(99)04930-8

\title{
INTEGRATION AND HOMOGENEOUS FUNCTIONS
}

\author{
JEAN B. LASSERRE
}

(Communicated by David H. Sharp)

\begin{abstract}
We show that integrating a (positively) homogeneous function $f$ on a compact domain $\Omega \subset R^{n}$ reduces to integrating a related function on the boundary $\partial \Omega$. The formula simplifies when the boundary $\partial \Omega$ is determined by homogeneous functions. Similar results are also presented for integration of exponentials and logarithms of homogeneous functions.
\end{abstract}

\section{INTRODUCTION}

We consider the integration of a continuous (positively) homogeneous function $f: R^{n} \rightarrow R$ on a compact domain $\Omega$ with boundary $\partial \Omega$. Using Euler's identity for homogeneous functions, Green's formula simplifies so that integrating $f$ on $\Omega$ reduces to integrating a (simply related) function on the boundary $\partial \Omega$. In the particular case where $\Omega:=\left\{x \in R^{n} \mid g_{i}(x) \leq a_{i}, i=1, \ldots, m\right\}$ and where the functions $g_{i}$ are each (positively) homogeneous of degree $p_{i}$, the formula is even simpler. Actually, an alternative proof that uses only Euler's formula is also outlined.

We thus extend to more general domains a result in [4] for integrating a homogeneous function on a convex polytope.

A potential application is the integration of arbitrary continuous functions on a compact set $\Omega$. Indeed, as the polynomials are sums of homogeneous polynomials, and are dense in $C(\Omega)$, the space of continuous functions on $\Omega$ with the sup norm, or even in $L_{1}(\Omega)$, one could approximate $\int_{\Omega} f d x$ by $\sum_{i} \int_{\Omega} P_{i} d x$, where the $P_{i}$ 's are homogeneous polynomials, and therefore use the previous result. This result could also be used in finite element methods for the integration of a poynomial on each individual volume element.

Finally, we also provide simple formulas for the integration of exponentials and logarithms of homogeneous functions. For instance, integrating $\log f$ on a convex polytope $\Omega$ reduces to integrating $\log f$ on $\partial \Omega$ and to computing the volume of $\Omega$.

\section{IntEgRATION OF A HOMOGENEOUS FUNCTION}

Let $f: R^{n} \rightarrow R$ be a real (positively) homogeneous function of degree $p$ (or in short, $f$ is $p$-homogeneous), i.e. $f(\lambda x)=\lambda^{p} f(x)$ for all $\lambda>0, x \in R^{n}$. For a (positively) $p$-homogeneous function that is continuously differentiable, Euler's

Received by the editors July 8, 1997.

1991 Mathematics Subject Classification. Primary 65D30.

Key words and phrases. Numerical integration in $R^{n}$, homogeneous functions.

(C)1999 American Mathematical Society 
formula states that

$$
p f(x)=\langle\nabla f(x), x\rangle \text { for all } x .
$$

2.1. On the Riemann-Green formula. We first treat the 2-dimensional case. Let $\Omega$ be a compact domain in $R^{2}$ with boundary $\partial \Omega$. Let $P(x, y)$ and $Q(x, y)$ be continuously differentiable on $\Omega$. Then, under some regularity conditions, the well-known Riemann-Green formula (cf. [5]) states that

$$
\iint_{\Omega}\left(\frac{\partial Q}{\partial x}-\frac{\partial P}{\partial y}\right) d x d y=\int_{\partial \Omega} P d x+Q d y
$$

This yields:

Lemma 2.1. Let $f(x, y)$ be a continuously differentiable p-homogeneous function on $\Omega$. Then

$$
(p+2) \iint_{\Omega} f d x d y=\int_{\partial \Omega} f(x, y)(x d y-y d x) .
$$

Proof. As $f$ is $p$-homogeneous, from (2.1)

$$
p f(x, y)=x \frac{\partial f}{\partial x}+y \frac{\partial f}{\partial y} \forall(x, y) \in \Omega .
$$

Let $P(x, y):=-y f(x, y)$ and $Q(x, y):=x f(x, y)$ for every $(x, y) \in \Omega$.

$$
\frac{\partial Q}{\partial x}-\frac{\partial P}{\partial y}=2 f(x, y)+x \frac{\partial f}{\partial x}+y \frac{\partial f}{\partial y}=(p+2) f(x, y) \forall(x, y) \in \Omega .
$$

Hence, applying the Riemann-Green formula (2.2) to $P$ and $Q$ yields

$$
(p+2) \iint_{\Omega} f d x d y=\int_{\partial \Omega} f(x, y)(x d y-y d x),
$$

the desired result.

When $f \equiv 1$ (i.e. $p=0$ ), one immediately retrieves the well-known formula

$$
\operatorname{area}(\Omega)=\frac{1}{2} \int_{\partial \Omega} x d y-y d x
$$

2.2. The general case. The previous result generalizes to $R^{n}$ as follows:

Lemma 2.2. Let $f: R^{n} \rightarrow R$ be a continuously differentiable p-homogeneous function and $\Omega$ a compact domain $R^{n}$ with boundary $\partial \Omega$. Then

$$
(n+p) \int_{\Omega} f d \omega=\int_{\partial \Omega}\langle\vec{M}, \vec{n}\rangle f(M) d \sigma
$$

where $\vec{n}$ is the unit outward-pointing normal to $\partial \Omega$.

Proof. With notation as in [6], let $X$ be the vector field $X:=\sum_{j} x_{j} \frac{\partial}{\partial x_{j}}$. From Proposition 2.3 in [6], we have

$$
\int_{\Omega} \operatorname{div}(X) f d \omega+\int_{\Omega} X f d \omega=\int_{\partial \Omega}\langle X, \vec{n}\rangle f d \sigma .
$$

Now, $\operatorname{div}(X)=n$ and from (2.1), $X f=p f$ so that the result follows.

Thus, integrating $f$ on $\Omega$ reduces to integrating $\langle\vec{M}, \vec{n}\rangle f$ on the boundary $\partial \Omega$. We next show that Lemma 2.2 further simplifies in the case where the boundary $\partial \Omega$ is determined by homogeneous functions. 


\section{BOUNDARY DETERMINED BY HOMOGENEOUS FUNCTIONS}

We now consider the special case where

$$
\Omega\left(\subset R^{n}\right):=\left\{x \in R^{n} \mid g_{i}(x) \leq a_{i}, i=1, \ldots, m\right\},
$$

and the functions $g_{i}$ are each continuously differentiable (positively) $p_{i}$-homogeneous functions, $i=1, \ldots, m$. We still assume that $\Omega$ is compact. Finally, let $\Omega_{i}:=\{x \in$ $\left.\Omega \mid g_{i}(x)=a_{i}\right\}$ so that $\partial \Omega=\bigcup_{i=1}^{m} \Omega_{i}$.

Theorem 3.1. Assume that $f$ is a continuously differentiable $q$-homogeneous function and the functions $g_{i}$ are each continuously differentiable and $p_{i}$-homogeneous, $i=1, \ldots, m$. Then

$$
(n+q) \int_{\Omega} f d \omega=\sum_{i=1}^{m} p_{i} a_{i} \int_{\Omega_{i}} \frac{f}{\left\|\nabla g_{i}\right\|} d \sigma .
$$

In particular, with $f \equiv 1$, one obtains

$$
n \times \operatorname{vol}(\Omega)=\sum_{i=1}^{m} p_{i} a_{i} \int_{\Omega_{i}}\left\|\nabla g_{i}\right\|^{-1} d \sigma .
$$

Proof. Lemma 2.2 applies so that

$$
(n+q) \int_{\Omega} f d \omega=\sum_{i=1}^{m} \int_{\Omega_{i}}\langle X, \vec{n}\rangle f d \sigma .
$$

Now, $\vec{n}=\left\|\nabla g_{i}\right\|^{-1} \nabla g_{i}$ on $\Omega_{i}$. Therefore, using (2.1), we have on $\Omega_{i}$

$$
\left\langle X, \nabla g_{i}\right\rangle=\left\langle x, \nabla g_{i}(x)\right\rangle=p_{i} g_{i}(x)=p_{i} a_{i},
$$

which yields the desired result.

Remark. When $\Omega$ is a convex polytope, i.e. $g_{i}(x)=\left\langle A_{i}, x\right\rangle$ and $p_{i}=1, i=1, \ldots, m$, then $\left\|\nabla g_{i}\right\|=\left\|A_{i}\right\|$ and thus (3.2) simplifies to

$$
(n+q) \int_{\Omega} f d \omega=\sum_{i=1}^{m} \frac{a_{i}}{\left\|A_{i}\right\|} \int_{\Omega_{i}} f d \sigma .
$$

In addition, one may iterate the process for $\int_{\Omega_{i}} f d \sigma$ (cf. [4] for more details).

An alternative proof. Interestingly enough, there is a direct proof that does not use Green's formula. It was used in [4] for the case where $\Omega$ is a convex polytope.

Write $a_{i}=b_{i}^{p_{i}}, i=1, \ldots, m$, and let

$$
h(b):=\int_{\Omega} f d \omega=\int_{\left\{g_{i}(x) \leq b_{i}^{p_{i}}, i=1, \ldots, m .\right\}} f d x .
$$

It is immediate that $h(\lambda b)=\lambda^{n+q} h(b)$, i.e. $h$ is $(n+q)$-homogeneous and continuously differentiable. In addition, one may show that

$$
\frac{\partial h}{\partial b_{i}}=p_{i} b_{i}^{p_{i}-1} \int_{\Omega_{i}} \frac{f}{\left\|\nabla g_{i}\right\|} d \sigma
$$

Hence, applying Euler's formula to $h$ yields

$$
(n+q) h(b)=\langle\nabla h(b), b\rangle=\sum_{i=1}^{m} p_{i} a_{i} \int_{\Omega_{i}} \frac{f}{\left\|\nabla g_{i}\right\|} d \sigma
$$

the desired result. 
Example. In $R^{2}$, consider the domain

$$
\Omega:=\left\{(x, y) \in R^{2} \mid x y \leq 1 ; x^{2}+y^{2} \leq R^{2} ; x, y \geq 0\right\}
$$

and its volume $\int_{\Omega} d \omega$ (i.e. $f \equiv 1$ is 0 -homogeneous).

Note that in (3.3), the coordinate axis boundaries of $\Omega$ do not contribute since the boundary values $a_{i}$ are zero in this case.

Both $g_{i}(x, y):=x y$ and $g_{2}(x, y):=x^{2}+y^{2}$ are 2-homogeneous. $\Omega_{1}:=\{(x, y) \in$ $\Omega \mid x y=1\}$ and $\Omega_{2}:=\left\{(x, y) \in \Omega \mid x^{2}+y^{2}=R^{2}\right\} .\left\|\nabla g_{1}(M)\right\|=\sqrt{x^{2}+y^{2}}$ so that on $\Omega_{1}$ we have $\left\|\nabla g_{1}(M)\right\|=\sqrt{\left(x^{4}+1\right) / x^{2}}$ and $d \sigma=\sqrt{\left(x^{4}+1\right) / x^{4}} d x$, which yields

$$
\int_{\Omega_{1}}\left\|\nabla g_{1}\right\|^{-1} d \sigma=\int_{a^{-1}}^{a} x^{-1} d x=2 \log (a)
$$

where $x=a$ is the solution to $x^{2}+y^{2}=R^{2}, x y=1$.

Similarly, $\left\|\nabla g_{2}\right\|=2 R$ on $\Omega_{2}$, so that

$$
\int_{\Omega_{2}}\left\|\nabla g_{2}\right\|^{-1} d \sigma=2 \times(1 / 2 R) \times(R \alpha)=\alpha,
$$

where $\alpha=\arctan \left(a^{-2}\right)$. Hence, Theorem 3.1 yields

$$
2 \operatorname{vol}(\Omega)=4 \log (a)+R^{2} \arctan \left(a^{-2}\right),
$$

i.e. $\operatorname{vol}(\Omega)=2 \log (a)+R^{2} \arctan \left(a^{-2}\right) / 2$. This can be retrieved directly.

If we now take $\Omega:=\{(x, y) \mid x y \leq 1 ; b \leq y \leq a ; x \geq 0\}$ with $a \geq b>0$, we get $\Omega_{2}:=\{(x, y) \in \Omega \mid y=a\}$ and $\Omega_{3}:=\{(x, y) \in \Omega \mid-y=-b\}$. Therefore,

$$
\int_{\Omega_{1}}\left\|\nabla g_{1}\right\|^{-1} d \sigma=\log \left(\frac{a}{b}\right) ; \int_{\Omega_{2}}\left\|\nabla g_{2}\right\|^{-1} d \sigma=\frac{1}{a} ; \int_{\Omega_{3}}\left\|\nabla g_{3}\right\|^{-1} d \sigma=\frac{1}{b},
$$

so that Theorem 3.1 yields

$$
2 \operatorname{vol}(\Omega)=2 \log \left(\frac{a}{b}\right)+a \frac{1}{a}-b \frac{1}{b}=2 \log \left(\frac{a}{b}\right),
$$

i.e. $\operatorname{vol}(\Omega)=\log (a / b)$, which is immediate to check.

\section{INTEGRATING EXPONENTIALS AND LOGARITHMS}

In this section, we consider exponentials and logarithms of homogeneous functions. Indeed, we show that integrating such functions on $\Omega$ reduces to integrating a related function on the boundary $\partial \Omega$.

4.1. Exponentials. We first consider the case of an exponential $e^{h(x)}$ where $h$ : $R^{n} \rightarrow R$ is $q$-homogeneous. The particular case where $h=\langle c, x\rangle$ was considered in [1] and [2] and was used to compute the number of integral points in a convex polytope. We have the following result.

Theorem 4.1. Let $\Omega$ be the convex polytope $\left\{x \in R^{n} \mid\left\langle A_{i}, x\right\rangle \leq a_{i}, i=1, \ldots, m\right\}$.

(a) Let $h: R^{n} \rightarrow R$ be q-homogeneous and continuously differentiable. Then, for every $k=0,1, \ldots$,

$$
(n+q k) \int_{\Omega} h^{k} e^{h} d \omega+q \int_{\Omega} h^{k+1} e^{h} d \omega=\sum_{i=1}^{m} \frac{a_{i}}{\left\|A_{i}\right\|} \int_{\Omega_{i}} h^{k} e^{h} d \sigma .
$$


(b) Let $h: R^{n} \rightarrow R$ be q-homogeneous, continuously differentiable and strictly positive on $\Omega$. Then, for every $k=1, \ldots$,

$$
(n-q k) \int_{\Omega} h^{-k} e^{h} d \omega+q \int_{\Omega} h^{-k+1} e^{h} d \omega=\sum_{i=1}^{m} \frac{a_{i}}{\left\|A_{i}\right\|} \int_{\Omega_{i}} h^{-k} e^{h} d \sigma .
$$

(c) If $p:=n / q$ is an integer, then

$$
q \int_{\Omega} h^{-p+1} e^{h} d \omega=\sum_{i=1}^{m} \frac{a_{i}}{\left\|A_{i}\right\|} \int_{\Omega_{i}} h^{-p} e^{h} d \sigma
$$

and $\int_{\Omega} h^{k} e^{h} d \omega, k=-p+1, \ldots,-1,0,1, \ldots$, can all be expressed as integrals on $\partial \Omega$. In particular,

$$
\begin{aligned}
q \int_{\Omega} e^{h} d \omega= & \sum_{i=1}^{m} \frac{a_{i}}{\left\|A_{i}\right\|} \int_{\Omega_{i}} e^{h}\left[h^{-1}-(p-1) h^{-2}+(p-1)(p-2) h^{-3}\right. \\
& \left.+\cdots+(-1)^{p+1}(p-1) ! h^{-p}\right] d \sigma
\end{aligned}
$$

(d) With $h:=\langle c, x\rangle$, and for every $\xi \in R^{n}$, we get

$$
\langle c, \xi\rangle \int_{\Omega} e^{\langle c, x\rangle} d \omega=\sum_{i=1}^{m} \frac{\left\langle A_{i}, \xi\right\rangle}{\left\|A_{i}\right\|} \int_{\Omega_{i}} e^{\langle c, x\rangle} d \sigma .
$$

Proof. Again, with $f:=h^{k} e^{h}$ and with the vector field $X:=\sum_{i} x_{i} \frac{\partial}{\partial x_{i}}$, Green's formula yields

$$
n \int_{\Omega} f d \omega+\int_{\Omega} X f d \omega=\int_{\partial \Omega}\langle X, \vec{n}\rangle f d \sigma .
$$

Using

$$
X f=\left(k h^{k-1}+h^{k}\right) e^{h} \sum_{i=1}^{n} x_{i} \frac{\partial h}{\partial x_{i}}=q\left(k h^{k}+h^{k+1}\right) e^{h}, \text { and }\langle X, \vec{n}\rangle=\frac{a_{i}}{\left\|A_{i}\right\|} \text { on } \Omega_{i},
$$

we obtain

$$
(n+q k) \int_{\Omega} h^{k} e^{h} d \omega+q \int_{\Omega} h^{k+1} e^{h} d \omega=\sum_{i=1}^{m} \frac{a_{i}}{\left\|A_{i}\right\|} \int_{\Omega_{i}} h^{k} e^{h} d \sigma,
$$

i.e. (4.1). Similarly, with $f:=h^{-k} e^{h}$ and similar arguments we obtain (4.2).

To get (c), just notice from (4.2) that $\int_{\Omega} h^{-n / q+1} e^{h} d \omega$ is expressed directly as an integral over $\partial \Omega$, which yields (4.3). Using (4.2) with $k:=n / q-1$ and (4.3), one obtains $\int h^{-n / q+2} e^{h} d \omega$ as an integral on $\partial \Omega$. Therefore, iterating and using (4.2) and (4.1), all the $\int_{\Omega} h^{k} e^{h} d \omega$, for $k:=-n / q+1, \ldots,-1,0,1, \ldots$, are expressed as integrals on $\partial \Omega$.

To get (d), apply Green's formula (4.6), but now with $X=\sum_{i} \xi_{i} \partial / \partial x_{i}$, so that $\operatorname{div}(X)=0$.

The formula (4.1) remains valid even with $h \equiv 0$ and $k=0$. With the convention $0^{0}=1$, (4.1) reduces to the volume formula (3.3) of $\Omega$.

Note that (4.5) was obtained in [1] using Stokes' formula, and a similar argument was used to show that it suffices to consider the vertices of the polyhedron. The same can be done using the above argument.

Indeed, let $\mathcal{H}_{i}$ be the $(n-1)$-dimensional affine variety that contains $\Omega_{i}$, and $\left\{u_{1}, \ldots, u_{n-1}\right\}$ an orthonormal basis of the associated vector space. $x \in \Omega_{i}$ can be 
written $x_{0}+\sum_{i=1}^{n-1} y_{i} u_{i}$ with $x_{0} \in \Omega_{i}$, arbitrary. On $\Omega_{i}$, consider the vector field $\sum_{1}^{n-1} \xi_{i} \partial / \partial y_{i}$. Green's formula yields

$$
\left\langle c, \sum_{i=1}^{n-1} \xi_{i} u_{i}\right\rangle \int_{\Omega_{i}} e^{\langle c, x\rangle} d \sigma=\sum_{j \neq i}\left\langle\xi, \vec{n}_{i}\right\rangle \int_{\Omega_{i j}} e^{\langle c, x\rangle} d \nu,
$$

where $\Omega_{i j}:=\Omega_{i} \cap \Omega_{j}$ and $\vec{n}_{i}$ (in the basis $\left\{u_{1}, \ldots, u_{n-1}\right\}$ ) is the unit outwardpointing normal to $\Omega_{i j}$. Obviously, the process can be repeated up to the 0 dimensional faces, i.e. the vertices of $\Omega$.

4.2. Logarithms. Consider now the function $\log f$ where $f: R^{n} \rightarrow R$ is continuously differentiable, $q$-homogeneous and strictly positive on $\Omega$.

Lemma 4.2. Let $\Omega \subset R^{n}$ be a compact domain with boundary $\partial \Omega$, and let $f$ : $R^{n} \rightarrow R$ be continuously differentiable, $q$-homogeneous and strictly positive on $\Omega$. Then

$$
n \int_{\Omega} \log f d \omega+q \times \operatorname{vol}(\Omega)=\int_{\partial \Omega}\langle\vec{M}, \vec{n}\rangle \log f(M) d \sigma .
$$

In addition, if $\Omega:=\left\{x \in R^{n} \mid\left\langle A_{i}, x\right\rangle \leq a_{i}, i=1, \ldots, m\right\}$, and $\Omega_{i}:=\{x \in$ $\left.\Omega \mid\left\langle A_{i}, x\right\rangle=a_{i}\right\}$, then

$$
n \int_{\Omega} \log f d \omega+q \times \operatorname{vol}(\Omega)=\sum_{i=1}^{m} \frac{a_{i}}{\left\|A_{i}\right\|} \int_{\Omega_{i}} \log f d \sigma .
$$

Proof. The proof is again the same as for the exponential. Note that with the vector field $X:=\sum_{j} x_{i} \partial / \partial x_{j}$, we have $X \log f=q$ and $\operatorname{div}(X)=n$, so that (2.5) reduces to $(4.9)$.

Hence, integrating $\log f$ on a convex polytope $\Omega$ reduces to integrating $\log f$ on the boundary $\partial \Omega$ and to computing the volume of the polyhedron.

\section{REFERENCES}

1. A. Barvinok, Computing the volume, counting integral points, and exponential sums, Discrete \& Computational Geometry 10 (1993), pp. 123-141. MR 94d:52005

2. M. Brion, Points entiers dans les polydres convexes, Ann. Sci. Ec. Norm. Sup., Srie IV, 21 (1988), pp. 653-663. MR 90d:52020

3. J.B. Lasserre, An analytical expression and an algorithm for the volume of a convex polyhedron in $R^{n}$, J. Optim. Theor. Appl. 39 (1983), pp. 363-377. MR 84m:52018

4. J.B. Lasserre, Integration on a convex polytope, Proc. Amer. Math. Soc. 126 (1998), 24332441. CMP 97:15

5. G. Strang, Introduction to Applied Mathematics, Wellesley-Cambridge Press, 1986. MR 88a:00006

6. M.E. Taylor, Partial Differential Equations: Basic Theory, Springer, New York, 1996. MR 98b:35002b

LAAS-CNRS, 7 Avenue du Colonel Roche, 31077 Toulouse Cédex 4, France

E-mail address: lasserre@laas.fr 